\title{
Differentiation of umbilical cord mesenchymal stem cells into hepatocytes in comparison with bone marrow mesenchymal stem cells
}

\author{
YA-BIN YU ${ }^{1,2^{*}}$, YAN SONG $^{2 *}$, YA CHEN $^{2}$, FENG ZHANG $^{1}$ and FU-ZHEN QI ${ }^{2}$ \\ ${ }^{1}$ Key Laboratory of Living Donor Liver Transplantation of Ministry of Public Health, Department of Liver Surgery, \\ The First Affiliated of Nanjing Medical University, Nanjing, Jiangsu 210029; ${ }^{2}$ Department of Hepatobiliary Surgery, \\ Huai'an First People's Hospital, Nanjing Medical University, Huai'an, Jiangsu 223300, P.R. China
}

Received December 27, 2017; Accepted May 22, 2018

DOI: $10.3892 / \mathrm{mmr} .2018 .9181$

\begin{abstract}
Mesenchymal stem cells (MSCs) are considered to be an ideal source for the cell therapy of end-stage liver diseases. Umbilical cord (UC)-MSCs can be obtained via a non-invasive procedure and can be easily cultured, making them potentially superior candidates for cell transplantation when compared with MSCs from other sources. In the present study, UC-MSCs were induced to differentiate into hepatocytes and were compared with bone marrow (BM)-MSCs for their hepatic differentiation potential. UC-MSCs showed significantly higher proliferation than BM-MSCs. Under hepatic induction, UC-MSCs and BM-MSCs could differentiate into hepatocytes. Reverse transcription-quantitative polymerase chain reaction (RT-qPCR) analysis revealed that a higher expression of the hepatocyte-specific genes albumin, cytochrome P450 3A4 (CYP3A4), tyrosine-aminotransferase, glucose-6phosphate, $\alpha 1$ antitrypsin and $\alpha$-fetoprotein was detected in differentiated UC-MSCs when compared with differentiated BM-MSCs. The results of ELISA and western blotting were in accordance with those of RT-qPCR. Theses results indicated that UC-MSCs had higher hepatic differentiation potential than BM-MSCs. Therefore, UC-MSCs may be advantageous over BM-MSCs for the treatment of end-stage liver disease.
\end{abstract}

Correspondence to: Dr Feng Zhang, Key Laboratory of Living Donor Liver Transplantation of Ministry of Public Health, Department of Liver Surgery, The First Affiliated of Nanjing Medical University, 300 Guangzhou Road, Nanjing, Jiangsu 210029, P.R. China

E-mail: zhangfeng2016zf@163.com

Dr Fu-Zhen Qi, Department of Hepatobiliary Surgery, Huai'an First People's Hospital, Nanjing Medical University, 1 West Huanghe Road, Huai'an, Jiangsu 223300, P.R. China

E-mail: qi.fuzhen@163.com

*Contributed equally

Key words: umbilical cord mesenchymal stem cell, bone marrow mesenchymal stem cell, hepatocyte

\section{Introduction}

With manifestations of liver failure, end-stage liver disease can be the termination of acute or chronic liver diseases. Hepatocyte transplantation is currently considered as a promising replacement resource for these diseases. However, transplantation is severely limited due to the serious shortage of liver donors, high expense, immunological rejection of the transplanted cells and requirement of long-term immunosuppression $(1,2)$. Therefore, it is necessary to find an alternative treatment to treat these serious liver injuries. Mesenchymal stem cells (MSCs) as therapeutic tools as they can be obtained with relative ease and expanded in culture, along with features of self-renewal and multidirectional differentiation have attracted considerable attention. Several studies have reported the isolation of MSCs from various sources, such as placenta, amniotic fluid, adipose tissue, bone marrow, and umbilical cord blood $(3,4)$. Bone-marrow mesenchymal stem cell (BM-MSC) which can be induced into hepatocyte is once the major source for MSC isolation. However, collecting bone marrow is an extremely invasive and painful procedure, and the proliferative ability, maximal cell lifespan and differentiation potential of BM-MSCs decrease with aging (5-8).

Umbilical cord (UC)-MSCs, an alternative source for MSC isolation, can be acquired by a non-invasive procedure and can be easily cultured, making them potentially superior candidates for cell transplantation compared with MSCs from other sources (9). Allogeneic transplantation of UC-MSCs can be applicable for cell therapy without immunological cross-reactivity (10). Also, it has been shown to express a low level of many liver-specific markers such as albumin (ALB), cytokeratins (CK) 18 and 19, $\alpha$-fetoprotein (AFP) (11).Therefore, UC-MSCs represent a prospective alternative cell source for hepatic disease therapies. To compare with their hepatic differentiation potential, both UC-MSCs and BM-MSCs were induced to differentiate into hepatocytes in this study.

\section{Materials and methods}

Isolation and culture of UC-MSCs. With the written informed consent of the donors and permission of the Institution 
Review Board and Human Ethics Committee of Huai'an First People's Hospital, Nanjing Medical University, fresh human umbilical cords were collected and stored in $0.9 \%$ normal saline containing $100 \mathrm{U} / \mathrm{ml}$ penicillin and $100 \mathrm{mg} / \mathrm{ml}$ streptomycin at $4^{\circ} \mathrm{C}$ after the delivery of the baby. There were ten donors involved in our experiment for the isolation of UC-MSCs and their age ranged from 22 to 36 years. The umbilical cord vessels were removed in $0.9 \%$ normal saline following disinfection in 75\% ethanol for $1 \mathrm{~min}$. The cord was cut into cubes of about $1 \mathrm{~cm}^{3}$. After removal of the supernatant fraction, the precipitate was rinsed with DMEM (Hyclone, Logan, UT, USA) and then centrifuged at $250 \mathrm{xg}$ for $5 \mathrm{~min}$. The tissue was digested with collagenase II (Gibco; Thermo Fisher Scientific, Inc., Waltham, MA, USA) at $37^{\circ} \mathrm{C}$ for $1 \mathrm{~h}$ and further treated with $0.25 \%$ trypsin (Hyclone) at $37^{\circ} \mathrm{C}$ for $0.5 \mathrm{~h}$. To neutralize the excess trypsin, fetal bovine serum (FBS) was added to the mesenchymal tissue. The dissociated mesenchymal cells were further dispersed with DMEM and counted using a hemocytometer. The live cells were then plated in a 6-well culture plate at a density of $1 \times 10^{6}$ cells per well (Cornings) and the medium was changed twice a week.

Isolation and culture of BM-MSCs. With the written informed consent of donors and permission of the Institution Review Board and Human Ethics Committee of Huai'an First People's Hospital, Nanjing Medical University, bone marrow samples were obtained and isolated as previously described (12). There were ten donors involved in our experiment for the isolation of BM-MSCs and their age ranged from 24 to 47 years. Six of them were males. A lymphoprep gradient was used to layer the bone marrow and then it was centrifuged at 2,000 rpm for $15 \mathrm{~min}$. Mononuclear cells were collected and resuspended in the growth medium. Cells were cultured in a 6-well tissue culture plate at a density of $1 \times 10^{6}$ cells per well and the medium was replaced after 3 days. The growth medium was changed twice a week. Cells were passaged with $0.25 \%$ trypsin when the cells reached $80-90 \%$ confluence.

Proliferative ability of UC-MSCs compared to BM-MSCs. UC-MSCs and BM-MSCs were digested with trypsin and counted after trypan blue staining when cells reached about $80 \%$ confluence during passages. Mean values of cell counts were calculated, and the mean population doubling of each passage was obtained according to the following formula: $\mathrm{PD}=(\operatorname{logNt}-\log \mathrm{N} 0) / \log 2$, where $\mathrm{Nt}$ is the harvested cell number and N0 is the initial cell number for each passage (13).

Flow cytometry analysis. The phenotype of MSCs was evaluated by flow cytometry using a flow cytometer (FACScan; BD Sciences, Shanghai, China). Native third passage UC-MSCs or BM-MSCs were trypsinized and suspended in PBS at a concentration of $1 \times 10^{7}$ cells $/ \mathrm{ml}$. Antibodies against human antigens CD13, CD105, CD34 and HLA-DR were purchased from BD Sciences. PE-as well as FITC-labeled mouse IgG were used as a negative control. The cells and antibodies were incubated at $4^{\circ} \mathrm{C}$ for $30 \mathrm{~min}$ and washed three times with PBS. Labeled cells were analyzed with the CELLQUEST Pro software (BD Sciences).
Osteogenic differentiation. After cells reached $\sim 80 \%$ confluence, the growth medium was changed to the osteogenic differentiation medium, consisting of DMEM-LG (Invitrogen; Thermo Fisher Scientific, Inc.) supplemented with 10\% FBS, $100 \mathrm{U} / \mathrm{ml}$ penicillin (Sigma-Aldrich; Merck KGaA, Darmstadt, Germany), $100 \mathrm{nM}$ dexamethasone (Sigma-Aldrich; Merck $\mathrm{KGaA}$ ), $100 \mu \mathrm{g} / \mathrm{ml}$ streptomycin (Sigma-Aldrich; Merck $\mathrm{KGaA}), 10 \mathrm{nM} \beta$-glycerophosphate (Sigma-Aldrich; Merck KGaA), 2 nM L-glutamine (Sigma-Aldrich; Merck KGaA) and $0.2 \mathrm{mM} \mathrm{L-ascorbate} \mathrm{(Sigma-Aldrich;} \mathrm{Merck} \mathrm{KGaA).} \mathrm{Cells}$ were cultured in the osteogenic differentiation medium for 21 days and the medium changed every 3 days. Differentiated cells were analyzed by alizarin red staining.

Adipogenic differentiation. Cells at passage 3 at a density of $1 \times 10^{4}$ cells $/ \mathrm{cm}^{2}$ were treated with adipogenic medium with medium changes twice weekly. Briefly, after cells reached $70 \%$ confluence, the medium was replaced with expansion medium consisting of L-DMEM supplemented with $10 \%$ FBS, $2 \mathrm{mM}$ IBMX (Sigma-Aldrich; Merck KGaA) and $5 \mu \mathrm{g} / \mathrm{ml}$ insulin solution (Sigma-Aldrich; Merck KGaA). After 3 weeks, the generation of lipid vacuoles were revealed by Oil Red O staining (Sigma-Aldrich; Merck KGaA).

Chondrogenic differentiation. Chondrogenic differentiation was carried out according to a previous method, the 4th passage cells were treated with chondrogenic medium for 3 weeks (A100701 StemPro Chondro DIFF kit; Gibco; Thermo Fisher Scientific, Inc.) (14). Medium changes were performed every 3 days, and chondrogenesis was assessed by immonohistochemical staining for type II collagen (KeyGen Biotech Co., Ltd., Nanjing, China).

Hepatic differentiation. According to the previous protocol (12), cells at passage 3 were deprived for 2 days in IMDM (Gibco; Thermo Fisher Scientific, Inc.) supplemented with $10 \mathrm{ng} / \mathrm{ml}$ bFGF (PeproTech, Inc., Rocky Hill, NJ, USA) and $20 \mathrm{ng} / \mathrm{ml}$ EGF (Peprotech) prior to induction using a two-step protocol. Differentiation was induced by treating MSCs with step-1 differentiation medium, consisting of IMDM supplemented with $20 \mathrm{ng} / \mathrm{ml}$ HGF (Peprotech), $10 \mathrm{ng} / \mathrm{ml} \mathrm{bFGF}$ and $0.61 \mathrm{~g} / \mathrm{ml}$ nicotinamide (Sigma-Aldrich; Merck KGaA) for 7 days, followed by treatment with step- 2 maturation medium, containing $20 \mathrm{ng} / \mathrm{ml}$ oncostatin M (OSM; Peprotech), $1 \mu \mathrm{mol} / 1$ dexamethasone (DEX; Sigma-Aldrich; Merck KGaA) and $50 \mathrm{mg} / \mathrm{ml}$ ITS. Medium was changed every 3 days.

Immunofluorescence analysis. Cells were washed twice with cold PBS and fixed in 4\% paraformaldehyde (KeyGen Biotech Co., Ltd.) in PBS for $30 \mathrm{~min}$ and permeabilized with PBS containing 0.1\% Triton X-100 (Sigma-Aldrich; Merck KGaA) for $20 \mathrm{~min}$. The samples were incubated with anti-human serum AFP antibody (Santa Cruz Biotechnology, Inc., Dallas, TX, USA), anti-human serum ALB antibody (Santa Cruz Biotechnology, Inc.), and anti-human serum cytochrome P450 3A4 (CYP3A4) antibody (Santa Cruz Biotechnology, Inc.), followed by incubation with second antibody conjugated with fluorescent phycobilioroteins, Dylight 594 and Alexa 488 goat anti-mouse immunoglobulin G (1:2,000; Sigma-Aldrich; Merck KGaA). Subsequently, 
the cells were stained with diamidinopheny-lindole (DAPI; Sigma-Aldrich; Merck KGaA) and observed under a fluorescence microscope (Olympus, Tokyo, Japan).

Reverse transcription-quantitative polymerase chain reaction (RT-qPCR) analysis. Total RNA was extracted from the cells using TRIzol reagent (Invitrogen; Thermo Fisher Scientific, Inc.) according to the manufacture's protocol. The cDNA templates were synthesized by oligo(dT) primer and PrimeScript RTase reverse transcriptase (Takara Biotechnology Co., Ltd., Dalian, China). The products were then subjected to RT-qPCR analysis using SYBR Green Mix (Applied Biosystems; Thermo Fisher Scientific, Inc.) with the specific primer pairs and conditions listed in Table I. The details of the thermocycling conditions were as follows: $95^{\circ} \mathrm{C}$ for $30 \mathrm{sec}$ (initial denaturation), followed by 40 cycles at $95^{\circ} \mathrm{C}$ for $5 \mathrm{sec}$ (exact denaturation) and $60^{\circ} \mathrm{C}$ for $30 \mathrm{sec}$ (primer annealing and PCR product elongation). The relative expression levels were determined using the comparative quantification cycle method, $2^{-\Delta \Delta C q}(15)$. The mRNA expression levels were normalized with GAPDH.

ELISA. After 1, 2, 3 and 4 weeks of differentiation, cells were washed twice with PBS and incubated for $2 \mathrm{~h}$ in DMEM-LG (5.5 mM glucose; Gibco; Thermo Fisher Scientific, Inc.). The medium was collected and stored at $-20^{\circ} \mathrm{C}$ until assayed. ALB and blood urea nitrogen (BUN) contents were measured using ELISA kit (Human Albumin ELISA kit ab108788 and Bmassay, Human Blood Ureas Nitrogen ELISA kit 27013; Abcam, Cambridge, UK) according to the manufacturer's instructions. TMB substrate was used with absorbance read at $450 \mathrm{~nm}$.

Western blot analysis. Total cellular protein was extracted using a cell lysis buffer. Protein were separated by electrophoresis and transferred to membranes. The membranes were blocked in blocking solution and incubated with mouse monoclonal Ab against AFP, ALB, glucose-6phosphate (G-6P), tryosine-aminotransferase (TAT), $\alpha 1$ antitrypsin ( $\alpha 1 \mathrm{AT}$ ) and CYP3A4 (1:200; Santa Cruz Biotechnology, Inc., AFP antibody sc-51506, ALB antibody sc-51515, G-6P antibody sc-373886, TAT antibody sc-365512, $\alpha 1$ AT antibody sc-73431, CYP3A4 antibody sc-53850) for $1 \mathrm{~h}$ at room temperature. After washing, the membranes were incubated for $2 \mathrm{~h}$ with horseradish peroxidase (HRP)-linked goat anti-mouse IgG (1:1,000; Santa Cruz Biotechnology, Inc., goat anti-mouse IgG-HRP, sc-2005). The membranes were rinsed for $10 \mathrm{sec}$ in substrate buffer to remove residual detergent. The protein bands were visualized by enhance chemiluminescence and the images were captured in X-ray film. Mouse monoclonal Ab against GAPDH (1:5,000; Santa Cruz Biotechnology, Inc., GAPDH antibody, sc-47724) was used as a housekeeping gene control. The protein quantities were determined relative to the internal optical densities of GAPDH reference standards using ImageJ software.

Statistical analysis. The results obtained from a typical experiment were expressed as the mean \pm standard deviation (SD). Group comparisons were made by Student's t-test and one-way analysis of variance. Multiple comparison between the groups was performed using the Student-Newman-Keuls method.

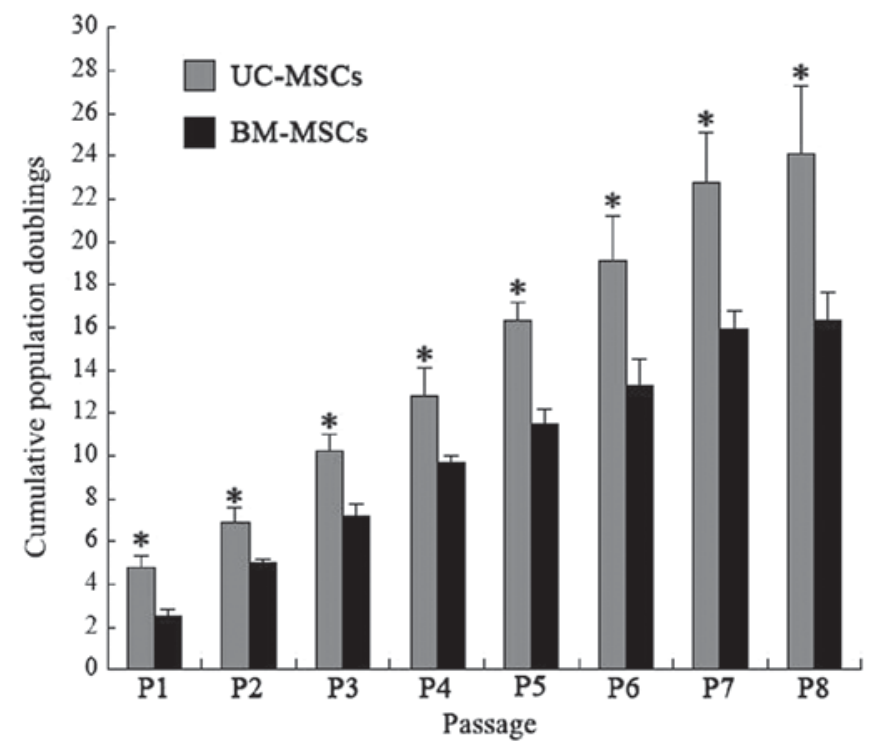

Figure 1. Cumulative population doublings. Cumulative population doublings of UC-MSCs and BM-MSCs from passage 1 to 8 were calculated according to cell counts. Each bar represents the mean \pm standard deviation $(n=3)$. ${ }^{*} \mathrm{P}<0.05$ vs. BM-MSCs. UC-MSCs, umbilical cord mesenchymal stem cells; BM-MSCs, bone marrow derived mesenchymal stem cells.

Statistical analysis was carried out using SPSS 16.0 software (SPSS, Inc., Chicago, IL, USA). A value of $\mathrm{P}<0.05$ was considered to indicate a statistically significant difference.

\section{Results}

Proliferative ability of UC-MSCs and BM-MSCs. UC-MSCs and BM-MSCs were respectively isolated from human umbilical cord and bone marrow. Both UC-MSCs and BM-MSCs were adherent, elongated and spindle-shaped. Cumulative population doublings of UC-MSCs and BM-MSCs were calculated from passage 1 to passage 8 . The cumulative population doublings of UC-MSCs at passage 8 was 23.8 while BM-MSCs was 16.5 (Fig. 1), indicating that UC-MSCs had greater proliferative ability than BM-MSCs.

Characterization of UC-MSCs and BM-MSCs. The cultured UC-MSCs expressed high levels of the MSC marker CD13, CD105 and did not express the hematopoietic marker CD34 and HLA-DR as a negative control (Fig. 2A). BM-MSCs also showed the similar expression of CD13, CD105, CD34 and HLA-DR (Fig. 2B). Under certain conditions, MSCs which are characterized as multipotent cells can differentiate into different cells. Positive staining of alizarin red indicated that UC-MSCs and BM-MSCs could differentiate into osteogenic cells (Fig. 3A2 and B2). Oil Red O staining showed that both UC-MSCs and BM-MSCs were positive for staining lipid droplets in the cytoplasm after adipogenic differentiation (Fig. 3A3 and B3). Both UC-MSCs and BM-MSCs cultured in the chondrogenic medium after differentiation did show immunohistochemical positive for type II collagen staining (Fig. 3A4 and B4).

Hepatogenic differentiation of UC-MSCs and BM-MSCs. To induce hepatic differentiation, cells were cultured in the hepatic 
Table I. Sequences of primers used for reverse transcription-quantitative polymerase chain reaction.

\begin{tabular}{|c|c|c|c|}
\hline Primer & Sequences (5'-3') & Fragment length (bp) & Annealing temperature $\left({ }^{\circ} \mathrm{C}\right)$ \\
\hline ALB & $\begin{array}{l}\text { F: TGCTTGAATGTGCTGATGACAGGG } \\
\text { R: AAGGCAAGTCAGCAGGCATCTCATC }\end{array}$ & 162 & 60 \\
\hline AFP & $\begin{array}{l}\text { F: GAAACCCACTGGAGATGAACAGTC } \\
\text { R: AAGTGGGATCGATGCAGGA }\end{array}$ & 190 & 60 \\
\hline TAT & $\begin{array}{l}\text { F: TGAGCAGTCTGTCCACTGCCT } \\
\text { R: ATGTGAATGAGGAGGATCTGAG }\end{array}$ & 359 & 60 \\
\hline G-6P & $\begin{array}{l}\text { F: GCTGGAGTCCTGTCAGGCATTGC } \\
\text { R: TAGAGCTGAGGCGGAATGGGAG }\end{array}$ & 349 & 60 \\
\hline CYP3A4 & $\begin{array}{l}\text { F: TGTGCCTGAGAACACCAGAG } \\
\text { R: GCAGAGGAGCCAAATCTACC }\end{array}$ & 202 & 60 \\
\hline$\alpha 1 \mathrm{AT}$ & $\begin{array}{l}\text { F: CTGGGACAGTGAATCGACAATGC } \\
\text { R: TCTGTTTCTTGGCCTCTTGGTG }\end{array}$ & 560 & 54 \\
\hline GAPDH & $\begin{array}{l}\text { F: AGAAGGCTGGGGCTCATTTG } \\
\text { R: AGGGCCATCCACAGTCTTC }\end{array}$ & 258 & 52 \\
\hline
\end{tabular}

ALB, albumin; CYP3A4, cytochrome P450 3A4; TAT, tyrosine-aminotransferase; G-6P, glucose-6phosphate; $\alpha 1$ AT, $\alpha 1$ antitrypsin; AFP, $\alpha$-fetoprotein; F, forward; R, reverse.

A
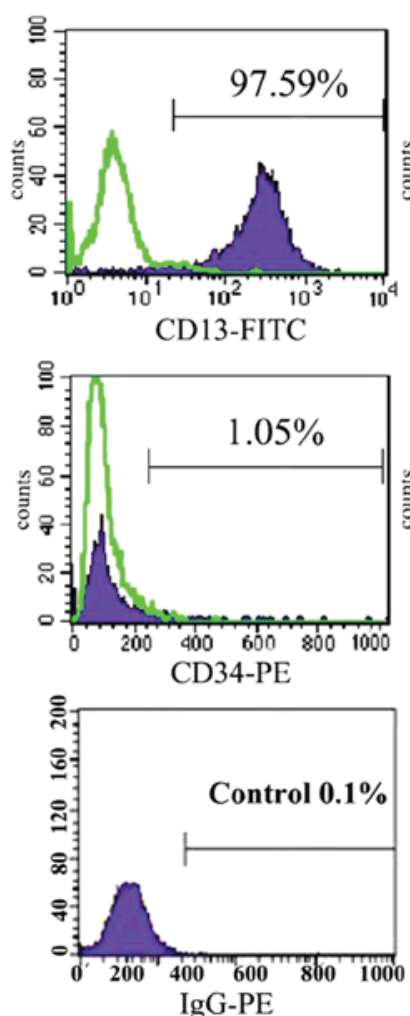

UC-MSCs
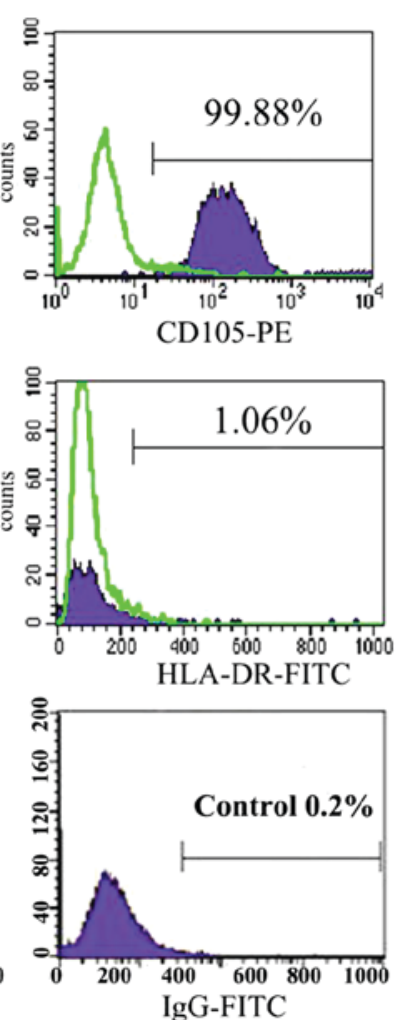

B

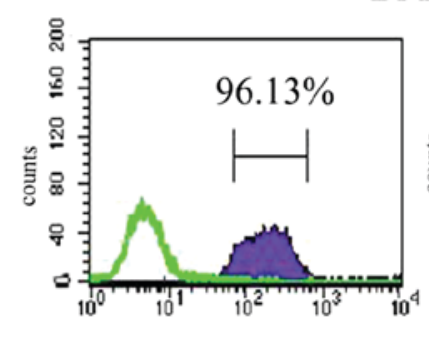

CD13-FITC

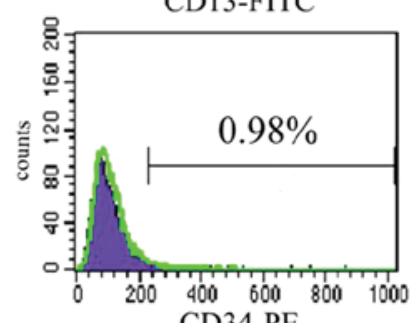

CD34-PE

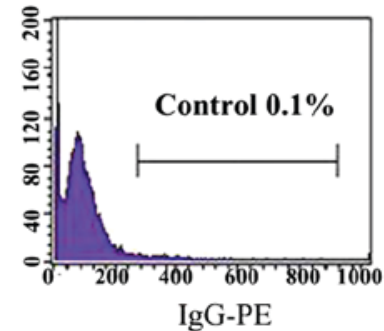

BM-MSCs

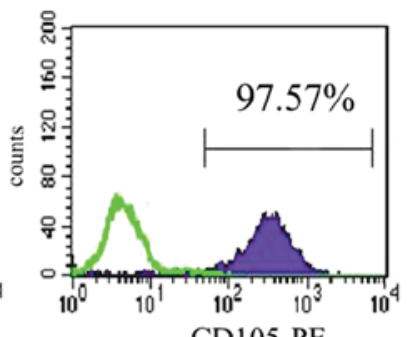

CD105-PE
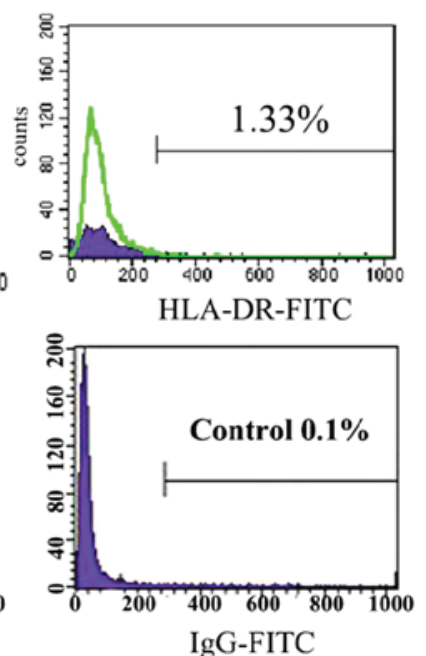

Figure 2. Analysis of MSC marker expression in (A) UC-MSCs and (B) BM-MSCs by flow cytometry. They were stained with PE- or FITC-conjugated antibodies. The respective immunoglobulin isotypes were used as negative controls and shown as open areas. MSCs, mesenchymal stem cells; UC-MSCs, umbilical cord-MSCSs; BM-MSCs, bone marrow derived-MSCs; PE, phycoerythrin; FITC, fluorescein isothiocyanate.

differentiation medium. Both UC-MSCs and BM-MSCs gradually formed the polygonal shape of hepatocytes with the appearance of aboundant granules in the cytoplasm (Fig. 4). We also detected hepatocyte-specific marker expression by immunofluorescene. UC-MSCs and BM-MSCs became positive for ALB, CYP3A4 and AFP after they were incubated in hepatic 
A

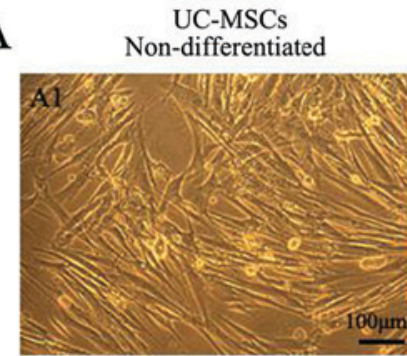

B

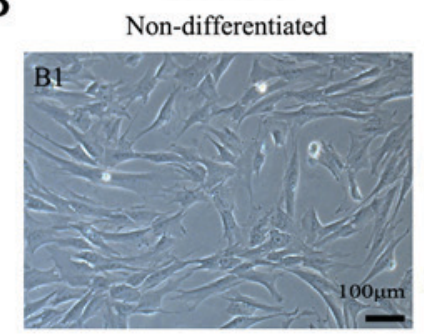

Alizarin red staining

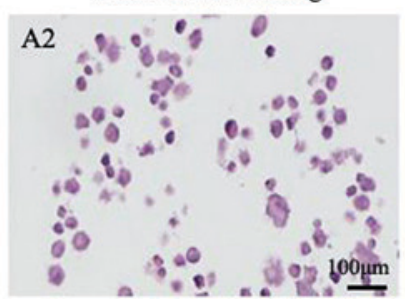

Alizarin red staining

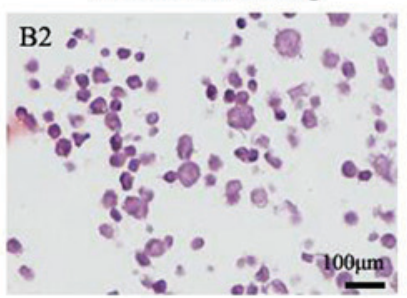

Oil red O staining

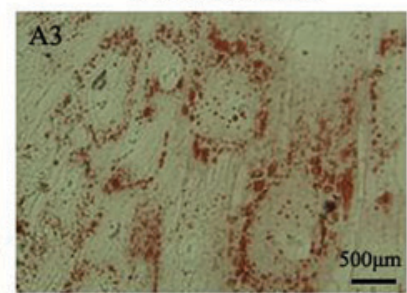

Oil red $\mathrm{O}$ staining

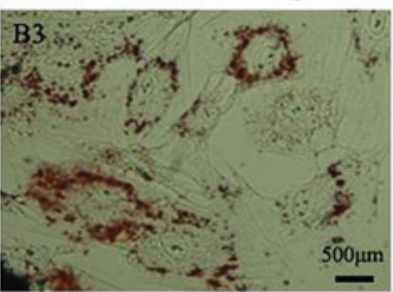

Type II collagen staining
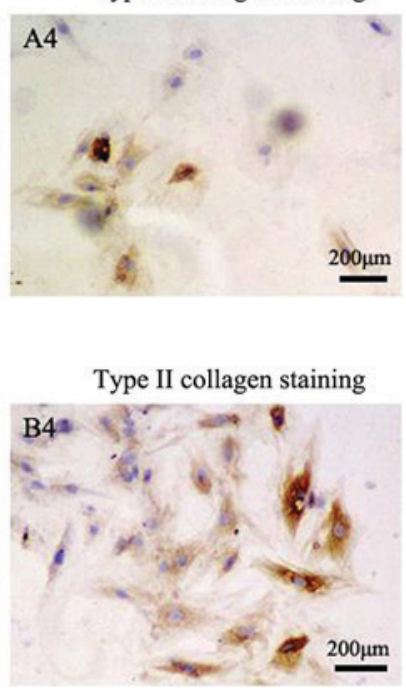

Figure 3. Detection of the differentiation potential of (A) UC-MSCs and (B) BM-MSCs. (A1) UC-MSCs and (B1) BM-MSCs were investigated for their differentiation capacity. (A2 and B2) Osteogenic differentiation was examined by alizarin red staining. Scale bars, $1-\mu \mathrm{m}$. (A3 and B3) Adipogenic differentiation was examined by Oil Red O staining. Scale bars, $500 \mu \mathrm{m}$. (A4 and B4) Cells were differentiated into chondrogenic cells and immunohistochemical stained positive for type II collagen. Scale bars, $200 \mu \mathrm{m}$. UC-MSCs, umbilical cord mesenchymal stem cells; BM-MSCs, bone marrow derived mesenchymal stem cells.

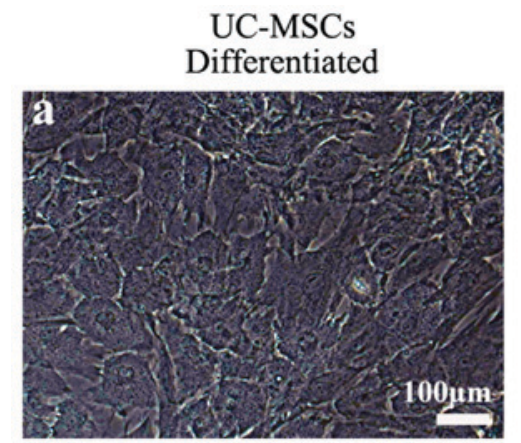

BM-MSCs

Differentiated

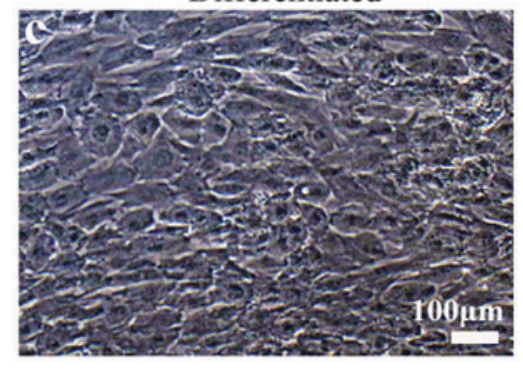

UC-MSCs

Un-differentiated

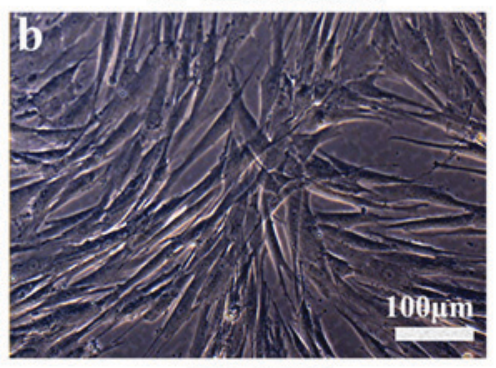

BM-MSCs

Un-differentiated

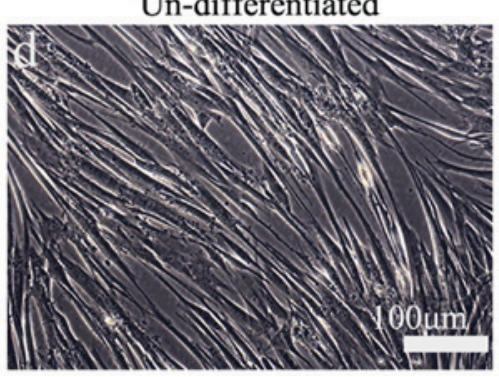

Figure 4. Hepatogenic differentiation in (a and b) UC-MSCs and (c and d) BM-MSCs. Hepatocyte-like cells differentiated from (a) UC-MSCs and (c) BM-MSCs following 4 weeks of induction. (b) UC-MSCs and (d) BM-MSCs cultured in growth medium were used as negative controls and were passaged when they reached 70-80\% confluence. Scale bars, $100 \mu \mathrm{m}$. UC-MSCs, umbilical cord mesenchymal stem cells; BM-MSCs, bone marrow derived mesenchymal stem cells.

differentiation medium for 4 weeks. UC-MSCs and BM-MSCs cultured in growth medium were as negative controls and they did not show positive signals for these markers (Fig. 5).

Hepatocytes-specific gene marker expression. After 4 weeks of induction, we examined the hepatic gene expression by RT-qPCR. The gene expression analysis of ALB, CYP3A4, TAT, G-6P, $\alpha 1$ AT from UC-MSCs group showed higher levels compared with BM-MSCs group while AFP showed lower expression. Hepatocyes expressed the six markers as a positive control (Fig. 6).

Measurement of secreted ALB and BUN. Both ALB and BUN are important indications of functional hepatocytes. From week 1 to 5 after hepatic differentiation, cell culture supernatants of UC-MSCs and BM-MSCs were collected every 
A
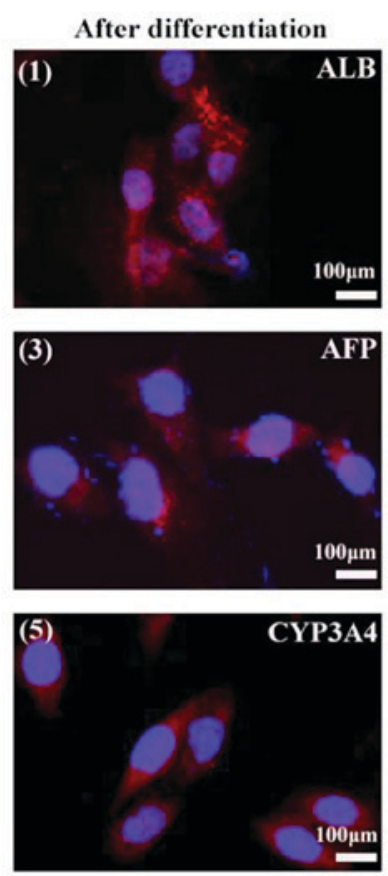

UC-MSCs
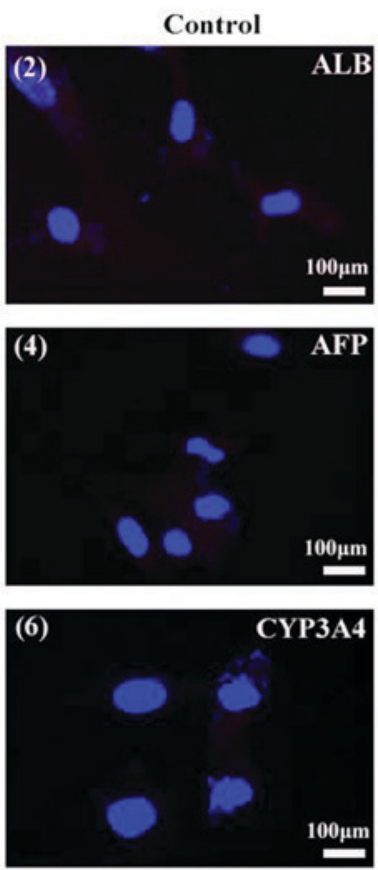

B
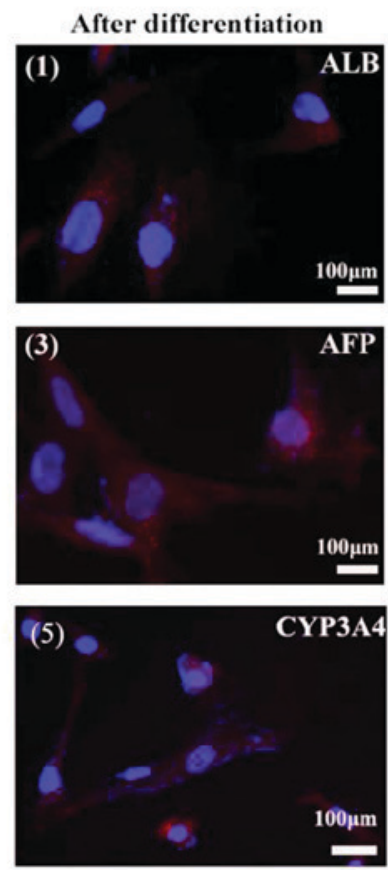

BM-MSCs
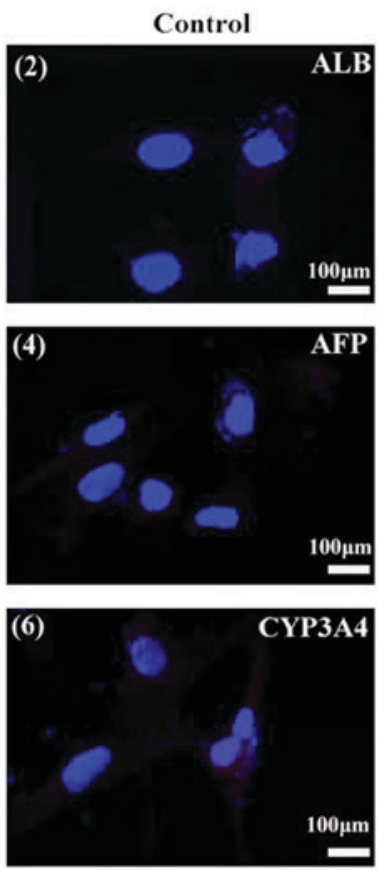

Figure 5. Immunofluorescent analysis of hepatocyte-specific proteins. (A) UC-MSCs and (B) BM-MSCs were examined for their expression of (A1 and B1) ALB, (A3 and B3) AFP, and (A5 and B5) CYP3A4 following hepatic differentiation for 4 weeks. (A2, 4 and 6, and B2, 4 and 6) Cells cultured in the growth medium were as negative controls. Scale bars, $100 \mu \mathrm{m}$. UC-MSCs, umbilical cord mesenchymal stem cells; BM-MSCs, bone marrow derived mesenchymal stem cells; ALB, albumin; AFP, $\alpha$-fetoprotein; CYP3A4, cytochrome P450 3A4.
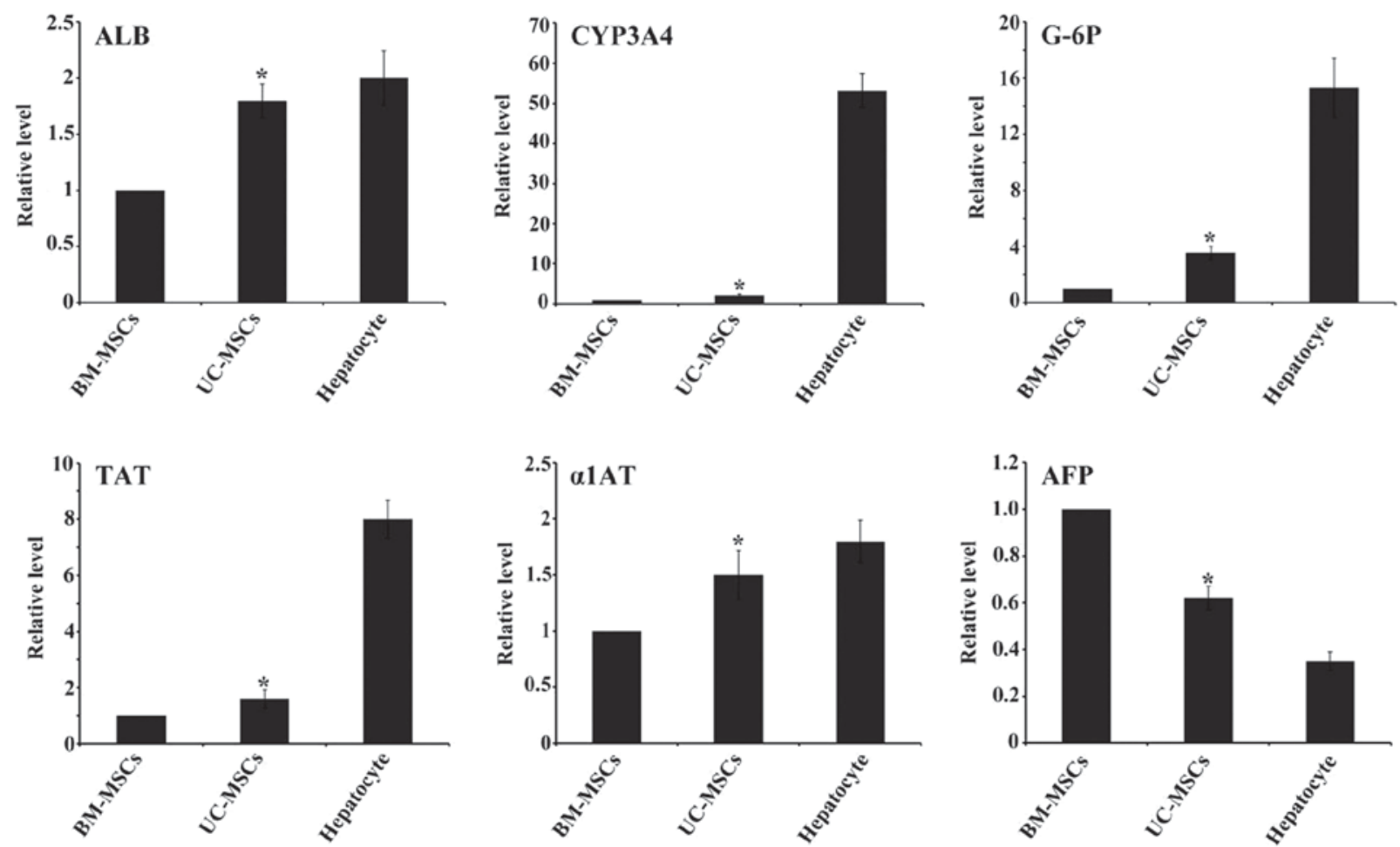

Figure 6. Expression levels of hepatocyte-specific genes ALB, TAT, CYP3A4, G-6P, $\alpha 1$ AT and AFP by reverse transcription-quantitative polymerase chain reaction. All of the data are presented as the mean \pm standard deviation $(n=3)$, and when compared with BM-MSCs, the fold induction for each gene induced by UC-MSCs was significant. "P<0.05 vs. BM-MSCs. UC-MSCs, umbilical cord mesenchymal stem cells; BM-MSCs, bone marrow derived mesenchymal stem cells; ALB, albumin; CYP3A4, cytochrome P450 3A4; TAT, tyrosine-aminotransferase; G-6P, glucose-6phosphate; $\alpha 1$ AT, $\alpha 1$ antitrypsin; AFP, $\alpha$-fetoprotein.

week and examined for the level of secreted ALB and BUN using ELISA kit. Both ALB and BUN levels were not detected before 1 week of induction. The ALB and BUN concentration increased from week 2 to week 5 after hepatic differentiation. 

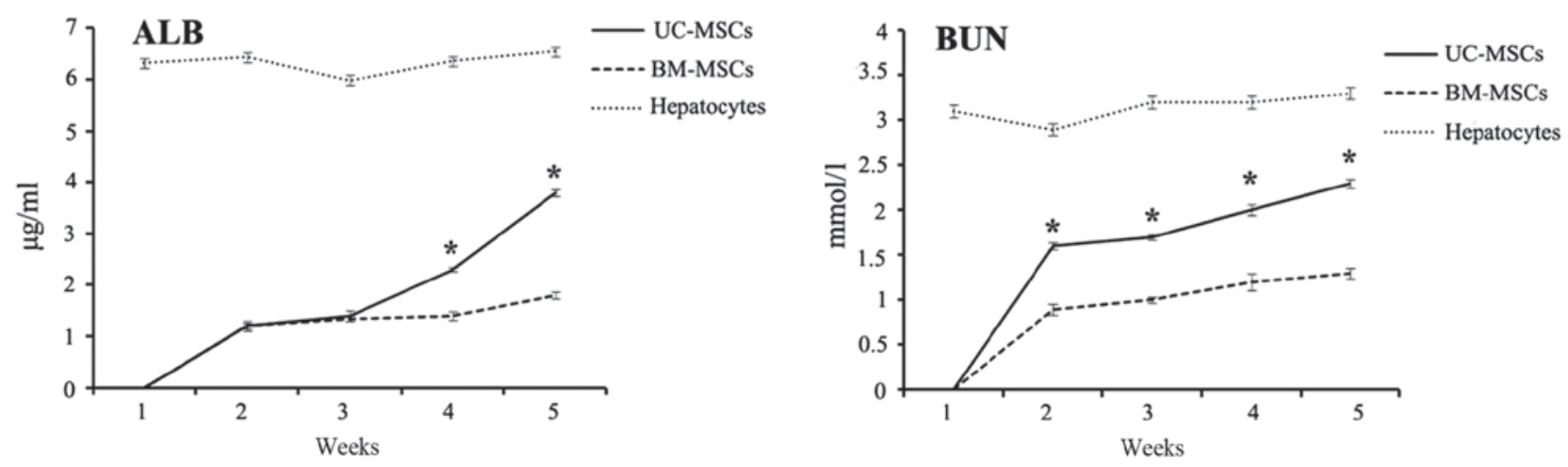

Figure 7. Detection of secreted albumin and blood urea nitrogen by ELISA. The ALB and BUN concentration of UC-MSCs and BM-MSCs culture supernatants collected on weeks 1,2,3,4 and 5 following hepatic differentiation were measured. The medium of hepatocyte was measured as a control. Each bar represents the mean \pm standard deviation $(\mathrm{n}=3)$. " $\mathrm{P}<0.05$ vs. BM-MSCs. UC-MSCs, umbilical cord mesenchymal stem cells; BM-MSCs, bone marrow derived mesenchymal stem cells; W, weeks; ALB, albumin; BUN, blood urea nitrogen.
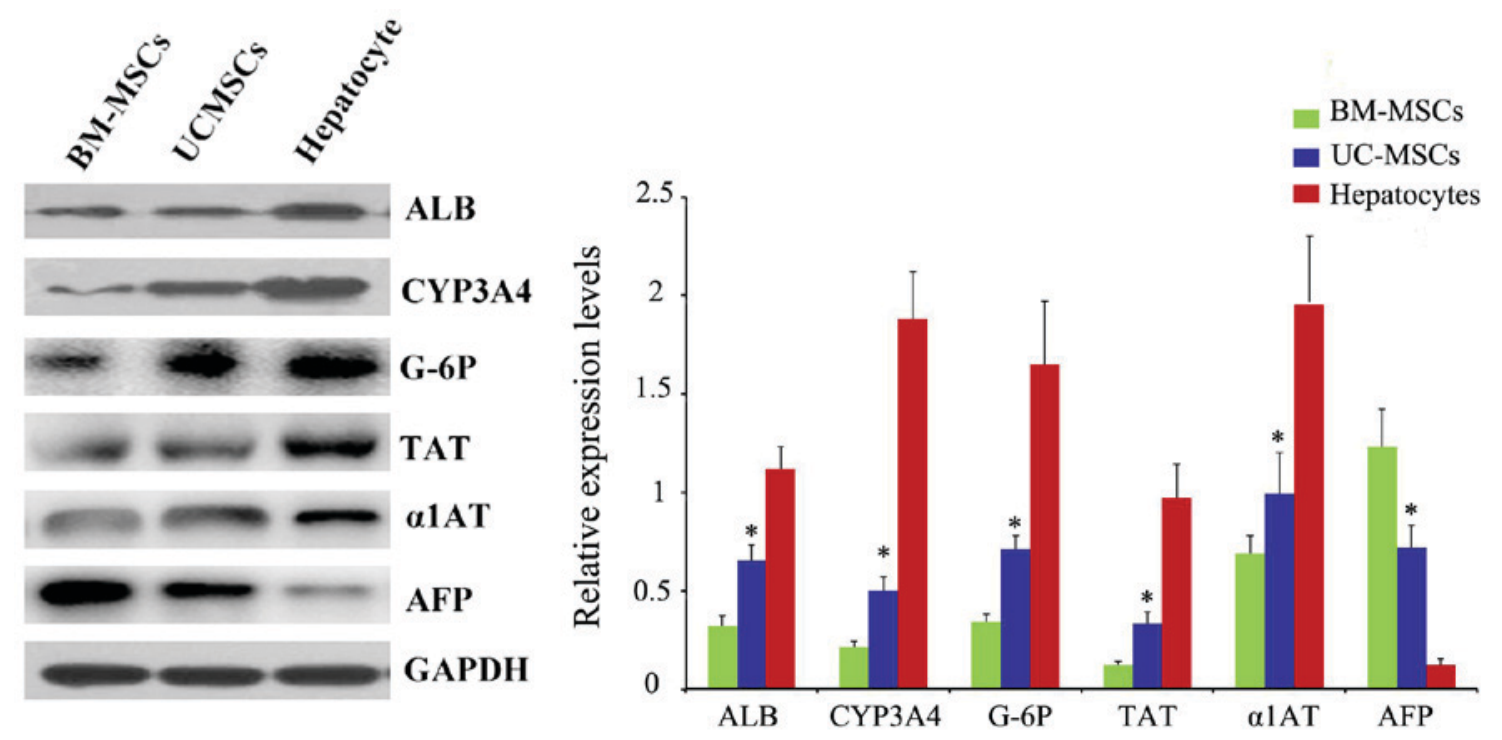

Figure 8. Protein levels of ALB, TAT, CYP3A4, G-6P, $\alpha 1$ AT and AFP were analyzed by western blot analysis. "P<0.05 vs. BM-MSCs. UC-MSCs, umbilical cord mesenchymal stem cells; BM-MSCs, bone marrow derived mesenchymal stem cells; ALB, albumin; CYP3A4, cytochrome P450 3A4; TAT, tyrosine-aminotransferase; G-6P, glucose-6phosphate; $\alpha 1 \mathrm{AT}, \alpha 1$ antitrypsin; AFP, $\alpha$-fetoprotein.

Compared with differentiated BM-MSCs, differentiated UC-MSCs secreted significantly more ALB and BUN after 4 weeks of induction $(P<0.05)$. Hepatocytes secreted ALB and BUN as a positive control (Fig. 7).

Western blot analysis of specific protein expression. We also examined the protein ALB, CYP3A4, TAT, G-6P, $\alpha 1 \mathrm{AT}$ and AFP after 4 weeks of induction by western blotting. Also, the result was in accordance with RT-qPCR (Fig. 8).

\section{Discussion}

MSCs are present in various tissues and are excellent candidates for cell therapy because of their capacity for self-renewal with a high proliferative capacity, multipotency, low immunogenicity. Our study showed that both UC-MSCs and BM-MSCs expressed high levels of the MSC markers CD13 and CD105, did not express the hematopoietic cell marker CD34 and HLA-DR, which were consistent with previous studies (16).
We have also examined other CD antigens of UC-MSCs and BM-MSCs through flow cytometry analysis. We found that both BM-MSCs and UC-MSCs expressed CD90, CD44, CD73 and CD59 (data not shown). Also, they did not express CD45, CD14, CD19 (data not shown).To illustrate their multipotent differentiation potential, both UC-MSCs and BM-MSCs were examined for their ability to undergo adipogenic, osteogenic and chondrogenic differentiation. However, UC-MSCs showed higher proliferation ability than BM-MSCs.

Previous studies showed that under certain conditions UC-MSCs and BM-MSCs could differentiate into hepatocyte $(12,17)$. In this study, UC-MSCs and BM-MSCs were cultured in the hepatic differentiation medium according to the protocol described by Lee et al (12). We found that, the two cells gradually began to form clusters and progressed toward the polygonal morphology of mature hepatocytes upon exposure to the differentiation medium. Also, they expressed hepatic protein markers, such as ALB, CYP3A4 and AFP. 
However, which cell has a higher hepatic differentiation efficiency remains unclear. This study first focused on comparing the function of hepatocytes differentiated from UC-MSCs and BM-MSCs. After 4 weeks of induction, we examined the hepatic gene expression by RT-qPCR. Compared to the gene expression of ALB, CYP3A4, TAT, G-6P and $\alpha 1 \mathrm{AT}$ in in the BM-MSCs group, the UC-MSCs group revealed higher level of these genes, whereas AFP exhibited lower expression. It indicated that the hepatocytes differentiated from UC-MSCs had a higher degree of maturity than BM-MSCs. ALB and BUN secretion were the important indication of functional hepatocytes. Both differentiated UC-MSCs and BM-MSCs began to secrete ALB and BUN on the 2 week of induction. Differentiated UC-MSCs secreted both ALB and BUN more than differentiated BM-MSCs after 4 weeks of induction. The protein levels of ALB, CYP3A4, TAT, G-6P, $\alpha 1 \mathrm{AT}$ and AFP were also examined by western blotting, and the results were in accordance with the RT-qPCR findings.

In conclusion, both UC-MSCs and BM-MSCs could be induced into hepatocytes under some conditions. Also, UC-MSCs had higher hepatic differentiation potential than BM-MSCs. Furthermore, without causing pain to donors, UC-MSCs can be obtained more easily compared with BM-MSCs, and the procedure avoids technical and ethical issues. UC-MSCs have a higher proliferation rate and are more primitive than BM-MSCs. Therefore, UC-MSCs has advantages over BM-MSCs for the treatment of end-stage liver disease.

\section{Acknowledgements}

Not applicable.

\section{Funding}

No funding was received.

\section{Availability of data and materials}

All data generated or analyzed during this study are included in this published article.

\section{Authors' contributions}

FZ and FZQ conceived and designed the experiments. YBY performed the experiments and wrote the manuscript. YS and YC performed the experiments and analyzed the data. All authors read and approved the final manuscript.

\section{Ethics approval and consent to participate}

The present study was performed with the permission of the Institution Review Board and Human Ethics Committee of Huai'an First People's Hospital, Nanjing Medical University (Jiangsu, China); written informed consent was obtained from all donors.

\section{Consent for publication}

Not applicable.

\section{Competing interests}

The authors declare that they have no competing interests.

\section{References}

1. Dhawan A, Puppi J, Hughes RD and Mitry RR: Human hepatocyte transplantation: Current experience and future challenges. Nat Rev Gastroenterol Hepatol 7: 288-298, 2010.

2. Zhou WL, Medine CN, Zhu L and Hay DC: Stem cell differentiation and human liver disease. World J Gastroenterol 18: 2018-2025, 2012

3. El-Tantawy WH and Haleem EN: Therapeutic effects of stem cell on hyperglycemia, hyperlipidemia, and oxidative stress in alloxan-treated rats. Mol Cell Biochem 391: 193-200, 2014.

4. In 't Anker PS, Scherjon SA, Kleijburg-van der Keur C, de Groot-Swings GM, Claas FH, Fibbe WE and Kanhai HH: Isolation of mesenchymal stem cells of fetal or maternal origin from human placenta. Stem Cells 22: 1338-1345, 2004.

5. Stenderup K, Justesen J, Clausen C and Kassem M: Aging is associated with decreased maximal life span and accelerated senescence of bone marrow stromal cells. Bone 33: 919-926, 2003.

6. Nishida S, Endo N, Yamagiwa H, Tanizawa T and Takahashi HE: Number of osteoprogenitor cells in human bone marrow markedly decreases after skeletal maturation. J Bone Miner Metab 17: 171-177, 1999.

7. Mueller SM and Glowacki J: Age-related decline in the osteogenic potential of human bone marrow cells cultured in three-dimensional collagen sponges. J Cell Biochem 82: 583-590, 2001.

8. Zhang H, Fazel S, Tian H, Mickle DA, Weisel RD, Fujii T and Li RK: Increasing donor age adversely impacts beneficial effects of bone marrow but not smooth muscle myocardial cell therapy. Am J Physiol Heart Circ Physiol 289: H2089-H2096, 2005.

9. Fan CG, Zhang QJ and Zhou JR: Therapeutic potentials of mesenchymal stem cells derived from human umbilical cord. Stem Cell Rev 7: 195-207, 2011.

10. Anzalone R, Lo Iacono M, Loria T, Di Stefano A, Giannuzzi P, Farina F and La Rocca G: Wharton's jelly mesenchymal stem cells as candidates for beta cells regeneration: Extending the differentiative and immunomodulatory benefits of adult mesenchymal stem cells for the treatment of type 1 diabetes. Stem Cell Rev 7: 342-363, 2011.

11. Prasajak P and Leeanansaksiri W: Developing a new two-step protocol to generate functional hepatocytes from Wharton's Jelly-derived mesenchymal stem cells under hypoxic condition. Stem Cells Int 2013: 762196, 2013.

12. Lee KD, Kuo TK, Whang-Peng J, Chung YF, Lin CT, Chou SH, Chen JR, Chen YP and Lee OK: In vitro hepatic differentiation of human mesenchymal stem cells. Hepatology 40: 1275-1284, 2004.

13. Kern S, Eichler H, Stoeve J, Klüter $H$ and Bieback K: Comparative analysis of mesenchymal stem cells from bone marrow, umbilical cord blood, or adipose tissue. Stem Cells 24: 1294-1301, 2006.

14. Wang HS, Shyu JF, Shen WS, Hsu HC, Chi TC, Chen CP, Huang SW, Shyr YM, Tang KT and Chen TH: Transplantation of insulin-producing cells derived from umbilical cord stromal mesenchymal stem cells to treat NOD mice. Cell Transplant 20: 455-466, 2011.

15. Livak KJ and Schmittgen TD: Analysis of relative gene expression data using real-time quantitative PCR and the 2(-Delta Delta C(T)) method. Methods 25: 402-408, 2001.

16. Dominici M, Le Blanc K, Mueller I, Slaper-Cortenbach I, Marini F, Krause D, Deans R, Keating A, Prockop Dj and Horwitz E: Minimal criteria for defining multipotent mesenchymal stromal cells. The International society for cellular therapy position statement. Cytotherapy 8: 315-317, 2006.

17. Campard D, Lysy PA, Najimi M and Sokal EM: Native umbilical cord matrix stem cells express hepatic markers and differentiate into hepatocyte-like cells. Gastroenterology 134: 833-848, 2008. 